\title{
Pre-operative Arterial Embolization of Symptomatic Giant Hemangioma of the Liver
}

Kayan M. ${ }^{1}$, Çetin M. ${ }^{1}$, Aktaş A. R. ${ }^{1}$, Yılmaz Ö. ${ }^{1}$, Ceylan E. ${ }^{1}$, Eroğlu H. E. ${ }^{2}$ ${ }^{1}$ Department of Radiology, Faculty of Medicine, Süleyman Demirel University, Isparta, Turkey;

${ }^{2}$ Department of General Surgery, Faculty of Medicine, Süleyman Demirel University, Isparta, Turkey

Received January 3, 2012; Accepted April 11, 2012.

Key words: Giant hemangioma - Embolization - Surgery - Symptomatic

Abstract: The cavernous hemangiomas of the liver are usually small sized and asymptomatic. Most of them are incidentally diagnosed and a very small portion requires therapy. Giant hemangioma can be symptomatic, and this condition is the indication for treatment. The striking complication of surgical treatment of cavernous hemangiomas is intra-operative bleeding. In this case, we aimed to demonstrate that the risk of intraoperative bleeding can be eliminated with the preoperative percutaneous trans-catheter arterial embolization technique.

Mailing Address: Dr. Mustafa Kayan, Department of Radiology, Faculty of Medicine, Süleyman Demirel University, Hızır Bey mah. 1556 sok. Yunus Evler Sitesi B-Blok, Kat:4 No:8, 32260 Isparta, Turkey; Phone: 050593535 39; e-mail: drkayan32@hotmail.com 


\section{Introduction}

The incidence of cavernous hemangiomas in the general population varies between $5-7 \%$. It is the most common benign tumor of the liver (Gandolfi et al., 1991). The origin of these tumors is not clearly known. Some authors believe that, the origin is hamartomatous, while other authors advocate that they are true vascular tumors (Giavroglou et al., 2003). Most of them are small and asymptomatic tumors, which are incidentally diagnosed and are characterized with good prognosis. Those more than $4 \mathrm{~cm}$ in size are referred to as giant hemangioma and they are rarely associated with abdominal pain. The symptomatic giant hepatic hemangiomas require treatment. Percutaneous trans-catheter arterial embolization (TAE) can be used or applied pre-operatively in the treatment of symptomatic hepatic cavernous hemangiomas (Stanley et al., 1983). We presented a case with hepatic hemangioma which had undergone surgical resection following percutaneous TAE.

\section{Case report}

The forty-year-old female patient referred to our hospital due to complaints of abdominal pain, lack of appetite and fatigue. Pain had increasing severity and disseminated from the epigastric region to the left hypochondrium. Physical examination of the patient revealed hepatomegalia. Hematological examination, hemoglobin, and other bleeding parameters appeared to be normal.

On the ultrasonographic (US) examination a hyperechoic giant mass lesion, originating from the left lobe of the liver and projecting to the epigastric region was detected. The lesion had regular borders and hypoechoic areas, which were suggestive of necrotic foci and cyst formations. Two homogenously hyperechoic lesions, which were 2 and $3 \mathrm{~cm}$ in size, were also observed in the right lobe of the liver. In order to clarify diagnosis, multi-phase contrast enhanced abdominal tomography (CT) was performed. On the dynamic CT examination, a giant exophytic mass lesion (dimensions were $13.5 \times 15 \times 20 \mathrm{~cm}$ ) was originating from the left lobe of the liver (Figure 1). It was extending from the epigastric region up to the sub-diapragmatic area. The lesion was compressing the spleen, stomach and left kidney downwards. After contrast administration the lesion showed peripheral nodular enhancement pattern in the arterial phase and in the portal and venous phase imaging progressive centripedal contrast filling was observed. On late phase images the lesion became iso-dense with the liver parenchyma. Lesions located in the right lobe of the liver had also similar contrast enhancement patterns. Those hepatic lesions were assessed as hemangioma based on ultrasonography and typical contrast enhancement pattern on CT.

The lesions located in right lobe of the liver were small in size and asymptomatic, and thus, radiological follow-up was prescribed. Surgical therapy was planned for the lesion in the left hepatic lobe since it was large, symptomatic and the pain did not respond to the analgesics.

Percutaneous TAE was planned for the cavernous hemangioma due to high risk of intraoperative bleeding. The patient was hospitalized and an abdominal digital 
subtraction arteriography (DSA) examination was performed via entry through the right main femoral artery using the Seldinger technique. On the DSA examination, arteria hepatica propria was originating from superior mesenteric artery. Selective catheterization of the left hepatic artery was performed via the superior mesenteric artery. The giant cavernous hemangioma located in the left hepatic lobe was imaged in arterial and parenchymal phases (Figure 2). The absence of the arteriovenous shunt was demonstrated with the venous phase. Later, the tip of the catheter was placed on the proximal aspect of the mass lesion, which also corresponds to the distal of left hepatic artery. Polyvinyl alcohol (Ivalon particles from Nycomed Ingenor, Paris, France) in various sizes was used in the embolization. The embolizing substances in various dimensions (45-150 $\mu \mathrm{m}, 150-250 \mu \mathrm{m}$ and 250-355 $\mu \mathrm{m})$ were sequentially administered and embolization of the capillary bed in the mass lesion was successfully performed, followed by embolization of all supplying arteries. On the post-procedure angiographic images, blood supply to the hemangioma was completely disappeared (Figure 3). In the following day, surgical resection of the hemangioma was successfully performed without bleeding complication.

\section{Discussion}

Hepatic hemangiomas are usually solitary and only $10 \%$ of all cases have multiple lesions. The hepatic cavernous hemangiomas are benign lesions, which usually have diameter of 1-3 cm. Eighty percent of the patients are female, the mean age ranges between $30-50$ years. Hemangiomas are usually asymptomatic. Other possible causes of abdominal pains, such as gallstones, gastroesophageal reflux disease or peptic ulcer disease should be pre-operatively ruled out. In symptomatic patient, it should be clearly emphasized that the hemangioma is responsible for the occurrence of the symptoms (Takayasu et al., 1990; Weimann et al., 1997; Gemer et al., 2004; Glinkova et al., 2004). Pain disappeared in our case following surgical resection.

Possible hematologic complications of giant cavernous hemangiomas include congestion, bleeding, thrombosis, infarction and Kasabah-Merritt syndrome. Spontaneous rupture is very rare. Giant hemangiomas may lead to compression on surrounding tissues, gastric outlet obstruction, biliary colic, and obstructive jaundice due to mass effects (Srivastava et al., 2001). Hepatomegalia was detected on physical examination and the laboratory examination was normal in our patient.

Cavernous hemangiomas can be correctly diagnosed with non-invasive radiologic imaging techniques. On US examination, hemangiomas usually are characterized as homogenous hyperechoic lesions with smooth borders. Heterogeneous and hypoechoic areas found within large sized cavernous hemangiomas are usually indicative of hemorrhage, thrombosis and infarction (Namimoto et al., 1997). Hepatic mass lesions can be imaged and diagnosed with a high rate of accuracy using sectional imaging methods. On non-contrast CT examination, hepatic hemangioma appears as a hypo-dense lesion, with smooth contours. On the multi-phase contrast enhanced abdominal CT examination, hepatic hemangioma has the following typical 


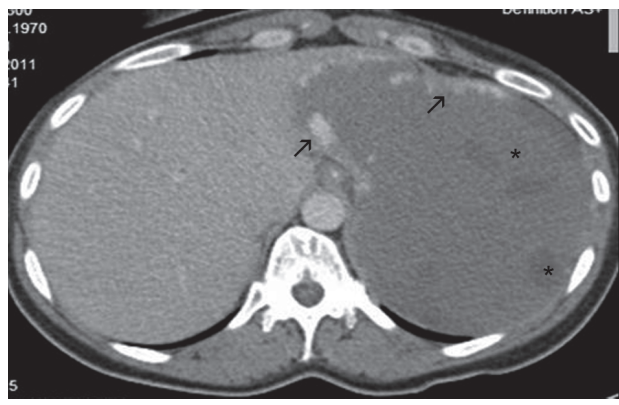

Figure 1 - Pre-embolization axial CT image shows the typical peripheral nodular contrast enhancement pattern (arrows) and some more hypo-dense areas (asterisk) in the giant cavernous hemangioma.

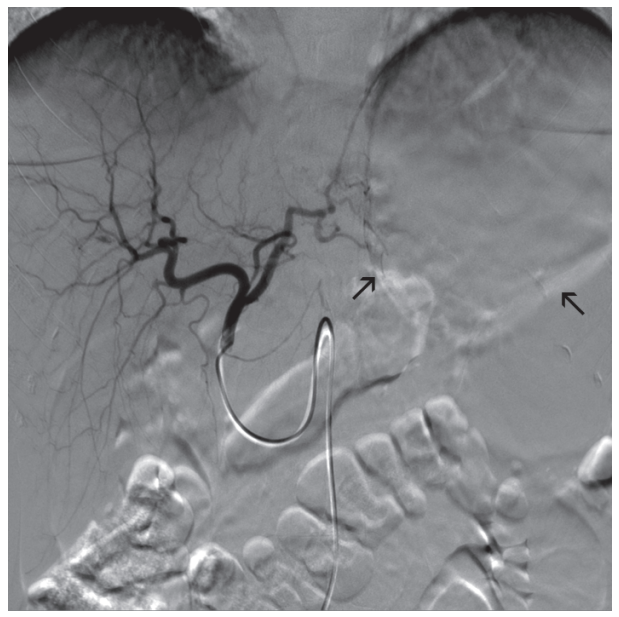

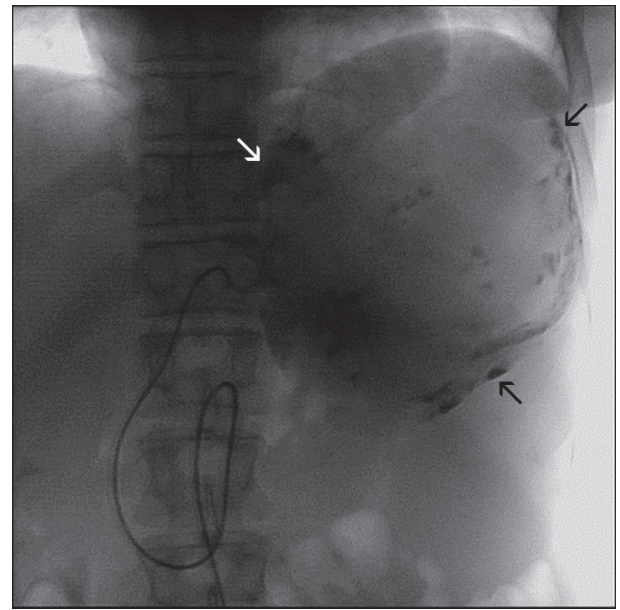

Figure 2 - Pre-embolization DSA image shows peripheral contrast accumulations (arrows) in the giant liver hemangioma.

Figure 3 - DSA demonstrate that the blood flow to the lesion (arrows) was completely disappeared just after the embolization.

findings: peripheral nodular pattern in arterial phase, progressive centripedal contrast uptake in portal and venous phase and iso-dense lesion, in comparison with the parenchyma, in the late stage. The multi-phase contrast enhanced CT findings of our case demonstrated typical contrast uptake pattern. The hypo-dense areas with no contrast uptake in any phase of the examination were considered as hemorrhagic or necrotic foci. MRI examination was not required since the CT and magnetic resonance imaging (MRI) have equal diagnostic value for hepatic hemangiomas.

In contrast to small-sized hemangiomas, giant hemangiomas are usually symptomatic. If the size of hepatic hemangioma is below $4 \mathrm{~cm}$ and the lesion is asymptomatic, treatment or follow-up is not required; however, if the size is over $4 \mathrm{~cm}$ and the lesion is symptomatic, treatment can be planned (Erdogan et al., 2007). Follow-up can also recommended in asymptomatic or mildly symptomatic giant 
hepatic hemangiomas but attention should be paid to complications (Srivastava et al., 2001).

Invasive procedures should be reserved for symptomatic hemangiomas, progressively growing hemangiomas, or the lesions bearing the risk of bleeding. The embolization procedure should be selectively performed, if possible, to involve all the branches supplying blood to the lesion. Various agents such as gelfoam, steel coils, polyvinyl alcohol and isobutyl cyanoacrylate can be used for embolization. In the embolization procedure, coils can be used for occluding the artery after the capillary bed is fulfilled with agents in various sizes. In the presence of arteriovenous shunt, particles cannot be used in order to prevent access of such materials to the systemic or portal system (Srivastava et al., 2001). In our case, the most common complications of the embolization did not occur, which include pain, pyrexia, leukocytosis and nausea.

The surgical resection criteria specified in the literature include progressive abdominal pain related with giant hemangioma, increase in size of the lesion, occurrence of complications and small hepatic lesions, which cannot be clearly discriminated from the hemangioma with a non-eliminated risk of malignancy. If possible, the non-invasive protective approach is recommended in the treatment of hemangiomas. For example, if the abdominal pain occurring due to giant hepatic hemangioma can be controlled with analgesics, no additional therapy is required (Srivastava et al., 2001).

The treatment of giant hepatic hemangiomas includes invasive approaches such as surgery and also less-invasive ones such as percutaneous TAE. The revascularization occurring over time causes failure of the method, although treatment with percutaneous TAE seems more attractive. Therefore, surgery is currently the most preferred option, in the treatment of giant hepatic hemangiomas. However, intraoperative bleeding is a significant and common complication of the surgery. Based on our case report, we demonstrated that the most important intra-operative complication of the surgical treatment of giant hepatic hemangiomas, the bleeding risk, can be eliminated with the use of the percutaneous TAE method.

In conclusion, the combination of percutaneous TAE and surgery is a safe procedure which eliminates the risk of intraoperative bleeding in the treatment of giant hepatic hemangioma, increases patient's comfort, and shortens the hospitalization period. 


\section{References}

Erdogan, D., Busch, O. R., Van-Delden, O. M., Bennink, R. J., Ten-Kate, F. J., Gouma, D. J., van-Gulik, T. M. (2007) Management of liver hemangiomas according to size and symptoms. J. Gastroenterol. Hepatol. 22, 1953-1958.

Gandolfi, L., Leo, P., Solmi, L., Vitelli, E., Verros, G., Colecchia, A. (1991) Natural history of hepatic haemangiomas: clinical and ultrasound study. Gut 32, 677-680.

Gemer, O., Moscovici, O., Ben-Horin, C. L., Linov, L., Peled, R., Segal, S. (2004) Oral contraceptives and liver hemangioma: a case-control study. Acta Obstet. Gynecol. Scand. 83, 199-201.

Giavroglou, C., Economou, H., loannidis, I. (2003) Arterial embolization of giant hepatic hemangiomas. Cardiovasc. Intervent. Radiol. 26, 92-96.

Glinkova, V., Shevah, O., Boaz, M., Levine, A., Shirin, H. (2004) Hepatic haemangiomas: possible association with female sex hormones. Gut 53, 1352-1355.

Namimoto, T., Yamashita, Y., Sumi, S., Tang, Y., Takahashi, M. (1997) Focal liver masses: Characterization with diffusion-weighted echo-planar MR imaging. Radiology 204, 739-744.

Srivastava, D. N., Gandhi, D., Seith, A., Pande, G. K., Sahni, P. (2001) Transcatheter arterial embolization in the treatment of symptomatic cavernous hemangiomas of the liver: a prospective study. Abdom. Imaging 26, 510-514.

Stanley, P., Grinnell, V., Stanton, R., Williams, K., Shore, N. (1983) Therapeutic embolization of infantile hepatic hemangioma with polyvinyl alcohol. AJR Am. J. Roentgenol. 141, 1041-1051.

Takayasu, K., Makuuchi, M., Takayama, T. (1990) Computed tomography of a rapidly growing hepatic hemangioma. J. Comput. Assist. Tomogr. 14, 143-145.

Weimann, A., Ringe, B., Klempnauer, J., Lamesch, P., Gratz, K. F., Prokop, M., Maschek, H., Tusch, G., Pichlmayr, R. (1997) Benign liver tumors: differential diagnosis and indications for surgery. World J. Surg. 21, 983-991. 